\title{
A Global Best Path Meteorological Data Gathering Algorithm for Wireless Sensor Networks
}

\author{
Yinghui Peng ${ }^{1}$, Bo Tang ${ }^{2}$, Yuan Xin ${ }^{1}$, Jin Wang ${ }^{2}$ and Jeong-Uk Kim ${ }^{3}$ \\ ${ }^{1}$ China Meteorological Administration, Research Centre for Strategic \\ Development, Beijing 100081, China \\ ${ }^{2}$ Jiangsu Engineering Center of Network Monitoring, Nanjing University of \\ Information Science \& Technology, Nanjing 210044, China \\ ${ }^{3}$ Department of Energy Grid, Sangmyung University, Seoul 110-743, Korea
}

\begin{abstract}
Sink mobility has been viewed as an important technique to improve network performance for wireless sensor networks (WSNs) such as energy consumption and balancing, network lifetime, throughput, end-to-end delay etc. Also, it can largely mitigate the hot spots near sink node as sink node moves randomly or autonomously. In many applications of WSNs, sensors are deployed in areas accessed by laid roads and sinks can be assembled on mobile devices like bus or handcart. In this paper, we propose a Global Best Path (GBP) data gathering algorithm based on wireless Sensor Networks with single Mobile Sink (GBP-MSSN). It aims at determining the best position for the single mobile sink and further using global sensors information to generate the best scheme to gather data from specified node. Generating of best scheme is conducted by GBP algorithm which can balance energy consumption among whole sensor networks and further prolong the network lifetime. Simulation results show that our GBP-MSSN algorithm outperforms conventional algorithms like LEACH, GAF, etc.
\end{abstract}

Keywords: Wireless sensor networks, global best path (GBP), mobile sink

\section{Introduction}

Wireless sensor networks (WSNs) are usually composed of hundreds or thousands of nodes which can sense, process and transmit their monitored data to remote sink node in a distributed, autonomous and multi-hop manner. Due to the intrinsic nature like selforganizing, infrastructure-less and fault-tolerance, WSNs have been widely used to many applications such as meteorological observation, military surveillance and battlefield, environmental monitoring, wildlife animal protection, home automation and health care etc., [1]. Nowadays, many communications protocols or algorithms are proposed such as LEACH [2], GAF [3], QAZP [4], TSA-MSSN [5] etc.

Since the huge amount of tiny and low cost sensors is deployed in an unattended manner (sometimes in very harsh environments), it is usually not possible to recharge them. Thus, how to utilize their limited energy resource in an efficient way so as to prolong network lifetime is the primary research challenge.

With the unceasingly thorough exploitation of nature, many regions like mountains, forest, boondocks which are inaccessible or hard to access by vehicles become easy to access by vehicles because of roads and other infrastructures. When some disasters like storm, fire, or pestilence disabled daily activities of humans in some areas, the roads and other infrastructures in these regions can be also workable. In many large-scale petrochemical industries, sensors used to gather air, water or other quality parameters are deployed in areas around the factory, and these areas can be easy to access by vehicles. 
In these scenarios, sensor fields always changes or are monitored for just several periods of time, thus it is not cheap or wise to build a good-sized and fixed base station (BS), namely sink. Mobile sink can solve these problems effectively. Furthermore, it outperforms fixed sink both in energy economization, delay decrease and lifetime prolongation of networks [6]. In addition, we argue that these mobile sinks can be carried by public surface transportation vehicles (e.g., cars, buses) that repeatedly pass fixed trajectories in sensor fields [7].

In this paper, we proposed a global best path data gathering algorithm for WSNs with single mobile sink (GBP-MSSN). Before gathering data from one node, GBP-MSSN needs to know the position set that the vehicle can arrive. For each position, the mobile sink computes locally and gets an estimation result of weight value by considering the global information, including nodes' positions and residual energy. GBP algorithm can tradeoff energy consumption and lifetime of networks by share energy cost among multiple nodes to avoid the hotspot phenomenon.

\section{Related Work}

Most of existing data gathering schemes or algorithms based on WSNs with single mobile sink are application-oriented and has their limitation or extra assumptions. Many studies focus on movement patterns of mobile sink and their main mentality is determining the best position for mobile sink previously based on the assumption that the mobile sink can arrive everywhere in sensor fields [8]. Actually, it is not practical in most real applications. In our proposed mechanism, we consider the sink's position based on its reachable position set, and this is more realistic for most applications.

In [9], an novel architecture of MSSNs in which sensors are sparsely deployed on both sides of roads or other routes is proposed. Based on this architecture, the authors further propose a transmission scheduling algorithm (TSA-MSSN). However, TSA-MSSN is only applicable to sparsely and along-roads deployed WSNs. In [10], network guided data collection (NGDC) is proposed as a novel mobility control solution. It achieves the tradeoff between delay and energy consumption. However, this method acquiesces that any position in sensor field is accessible, and actually, it is not practical in real applications. It also asks for mass storage to the DG nodes.

Authors in [11] propose an energy-aware routing to maximize lifetime in wireless sensor networks with mobile sink. It achieves maximum lifetime by solving optimally two joint problems: sojourn time at different position and energy-efficient routing. However, it suffers the same problem as NGDC has, namely assuming that the whole sensor field is accessible.

Authors in [12] put forward a wireless sensor network with mobile relays which have more energy than the static sensors. The cost to build and maintain such a device is not cheap. And this method needs some of nodes to aware of the position of the relay in realtime, as a result, these nodes suffer extra energy consumption.

\section{Our Proposed GBP-MSSN Algorithm}

\subsection{Main Idea of GBP-MSSN}

GBP-MSSN is a highly centralized algorithm and it consists of two parts. Firstly, it utilizes global best path algorithm (GBP) to generate a best scheme for each position in the available position set. Secondly, it chooses the best position with minimum weight to gather result data from specified node. The weight can be computed via equation (1) below in which $N E_{C}(i, j)$ stands for the normalized total energy consumption of node $\mathrm{i}$ in per transmission to node $\mathrm{j}, N E_{\text {rest }}(i)$ stands for the normalized rest energy of node $\mathrm{i}$.. 


$$
W(i)=\frac{N E_{C}(i, j)}{N E_{\text {rest }}(i)}
$$

\subsection{Procedure of GBP-MSSN}

There are four phases for mobile sink to gather data from a specified node. (1) Phase 1: Input available position set

One problem is how to get the available position set. There are two methods to get available position set. One is traveling the available position and writing down each position. The other one is computing the possible positions based-on precise map.

Another problem is how to transform the continuous position lines into discrete position points. A road is commonly a continuous line and there are unnumbered position points on it. It is not necessary to write down each point on the road. We can divide the road into many sub segments and write down endpoints of each of them.

(2) Phase 2: Scheme generating

For each position at phase 1, we compute the energy cost to gather data from a specified source node. We consider the network which has $\mathrm{N}$ nodes identified by 1 to $\mathrm{n}$ and the $\mathrm{n}+1$ node is the mobile sink. We denote position set as $P\left\{p_{1}, p_{2}, p_{3} \ldots p_{l}\right\}$.

In equations (2)-(6), Flag (i) stands for node with its best path, SCost $(i, j)$ refers to normalized total energy cost (include receiving and transferring) for node $\mathrm{j}$ to transmit 2000 bits data to node i, SBC (i) refers to normalized total energy cost, SRE (i) refers to normalized rest energy of node $\mathrm{i}, A(i)$ refers to evaluation value of the current best path, $A N(i, j)$ refers to weight between node $\mathrm{i}$ and node $\mathrm{j}$ and $\operatorname{BestA}(g)$ refers to optimum evaluation value for position $p_{g}$;

$$
\begin{gathered}
A(i)=\frac{\operatorname{SBC}(i)}{\operatorname{SRE}(i)} \quad(1 \leq i \leq n) \\
A N(i, j)=\frac{\operatorname{SCost}(i, j)}{\operatorname{SRE}(j)} \quad(1 \leq i \leq n, 1 \leq j \leq n) \\
A(k)=\operatorname{Min}\{A(i) \mid \text { Flag } \quad(i)=0\} \quad(1 \leq i, k \leq n) \\
A(i)=A(k)+A N(k, i) \quad(1 \leq i, k \leq n) \\
\operatorname{BestA}(g)=A(i) \quad(1 \leq i \leq n, 1 \leq g \leq l)
\end{gathered}
$$

After getting the optimum gathering scheme with the best weight value Besta $(g)$ for position $p_{g}$, we further compare it with the best weight value of last position, namely Besta $(g-1)$, and choose the scheme with lower weight value as the current best scheme. After $\mathrm{j}-1$ times of comparisons, we can get the final best scheme with the best position $p_{\text {best }}$ and the lowest weight value.

(3) Phase 3: Vehicle orientation \& order broadcast

Mobile sink first moves to position $p_{\text {best }}$, then it broadcasts orders to start a query.

(4) Phase 4: Data transmission 
When all related nodes receive orders from sink, they execute these orders. Channel assigning in MAC layer of this procedure can be conducted by time division multiple access (TDMA) protocols.

\section{Performance Evaluation}

As is shown in Figure 1, average residual energy of the network using GBP-MSSN decreases most slowly and steadily. That stands for GBP-MSSN achieves balancing energy consumption among all nodes of the network.

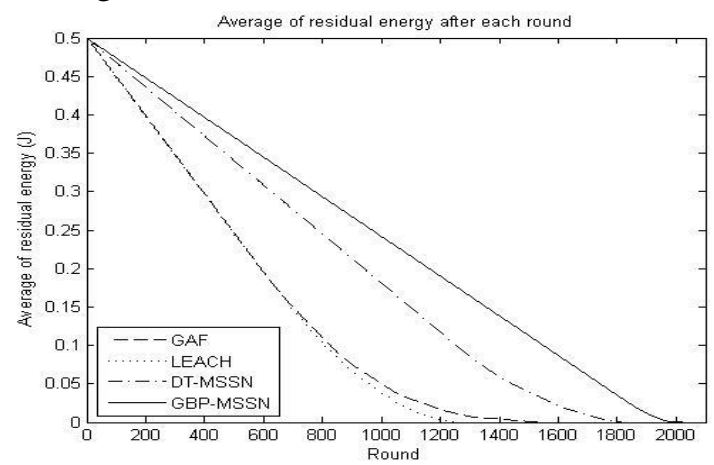

Figure 1. Average Residual Energy for Different Algorithms

Table 1 shows us the network lifetime using different algorithms. In general, DTMSSN and GBP-MSSN consume more energy than the others. GBP-MSSN can further improve direct transmission based on MSSN. Figure 2 shows the detail number of nodes alive after each round, and the result is consistent with the analysis above.

Table 1. Network Lifetime using Different Algorithms

\begin{tabular}{|l|l|}
\hline \multicolumn{1}{|c|}{ Algorithm } & \multicolumn{1}{c|}{ Network Lifetime } \\
\hline DT-MSSN & 1302 \\
\hline LEACH & 1060 \\
\hline GAF & 624 \\
\hline GBP-MSSN & 1844 \\
\hline
\end{tabular}

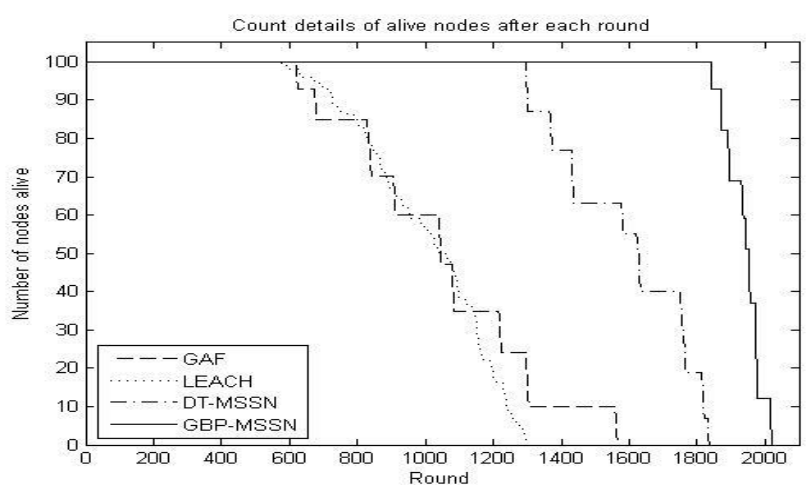

Figure 2. Number of Nodes Alive for Different Algorithms

\section{Conclusions}

This paper exploited a novel and efficient data gathering algorithm called GBP-MSSN which is based on wireless Sensor Networks with single Mobile Sink (MSSN). To deal with large-scale networks, we recommend using GBP combined with other clustering 
protocols like LEACH, HEED, GAF, etc. Specific approach is that first using clustering algorithm to gather data and store the compressed data locally in cluster head nodes, then using GBP-MSSN algorithm to gather data from cluster head nodes. Simulation results reveal that GBP-MSSN outperforms other algorithms.

\section{Acknowledgements}

It was supported by the "Public Policy Research of New media transmission for Meteorological Information", which is one of China Meteorological Administration soft science key research projects in 2014. It was also supported by the Industrial Strategic Technology Development Program (10041740) funded by the Ministry of Trade, Industry and Energy Korea. Prof. Jeong-Uk Kim is the corresponding author.

\section{References}

[1] Z. Pei, Z. Deng, B. Yang and X. Cheng, "Application-oriented wireless sensor network communication protocols and hardware platforms: A survey", IEEE International Conference on Industrial Technology, (2008) April 21-24.

[2] W. R. Heinzelman, A. Chandrakasan and H. Balakrishnan, "Energy-efficient communication protocol for wireless microsensor networks", Proceedings of the 33rd Annual Hawaii International Conference on System Sciences, (2000) January.

[3] S. Roychowdhury and C. Patra, "Geographic Adaptive Fidelity and Geographic Energy Aware Routing in Ad Hoc Routing", International Conference of ACCTA, (2010) August 3-5.

[4] S.-T. Cheng and T.-Y. Chang, "An adaptive learning scheme for load balancing with zone partition in multi-sink wireless sensor network", Expert Systems with Applications, vol. 39, (2012), pp. 9427-9434.

[5] L. Song and D. Hatzinakos, "Architecture of Wireless Sensor Networks with Mobile Sinks: Sparsely Deployed Sensors", IEEE Trans. on Vehicular Technology, vol. 56, no. 4, (2007), pp. 1826-1836.

[6] Y. Yun and Y. Xia, "Maximizing the Lifetime of Wireless Sensor Networks with Mobile Sink in DelayTolerant Applications", IEEE Trans. on Mobile Computing, vol. 9, no. 9, (2010), pp. 1308-1318.

[7] C. Konstantopoulos, G. Pantziou, D. Gavalas, A. Mpitziopoulos and B. Mamalis, "A Rendezvous-Based Approach Enabling Energy-Efficient Sensory Data Collection with Mobile Sinks”, IEEE Transactions on Parallel and Distributed Systems, vol. 23, no. 5, (2012), pp. 809-817.

[8] Y. Bi, L. Sun, J. Ma, N. Li, I. Khan and C. Chen, "Hums: An autonomous moving strategy in data gathering sensor networks", Journal on Wireless Communication and Networking, (2007).

[9] M. Ma and Y. Y Yang, "SenCar: An energy-efficient data gathering mechanism for large-scale multihop sensor networks", IEEE Trans. on Parallel and Distributed Systems, vol. 18, no. 10, (2007), pp. 14761488.

[10] J. Luo and J. Hubaux, "Jiont mobility and routing for lifetime elongation in wireless sensor networks", INFOCOM, (2005), pp. 1735-1746.

[11] L. Song and D. Hatzinakos, "Architecture of Wireless Sensor Networks with Mobile Sinks: Sparsely Deployed Sensors", IEEE Trans. on Vehicular Technology, vol. 56, no. 4, (2007), pp. 1826-1836.

[12] J. Rao and S. Biswas, "Data harvesting in sensor networks using mobile sinks", IEEE Wireless Communications, vol. 15 , no. 6, (2008), pp. 63-70.

\section{Authors}

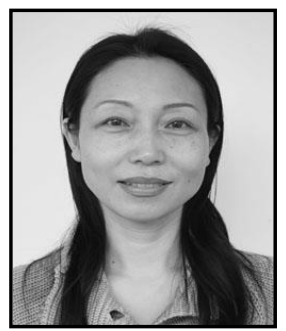

Yinghui Peng, received her B.S. degree from Nanjing University of Information Science and Technology, China. She is now working at the Development Research Center of China Meteorological Administration as an associate researcher and director of the strategy study and information supporting branch. Her research interests mainly include the study of climate change and information propagation etc. 


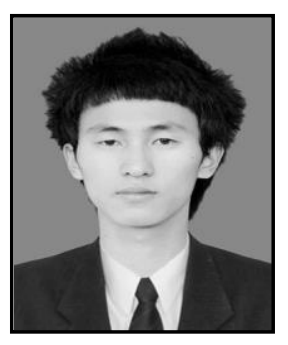

Bo Tang, received the B.S. degree from Nanjing University of Information Science \& Technology, China in 2011. Now he is studying for a M.S. degree in his Alma Mater. His research interests include wireless sensor networks, routing protocol and algorithm design, optimization for existed protocols and algorithms.

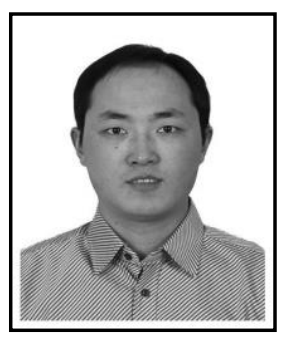

XinYuan, received the master degree from management department of Nan Jing University. He is now working in the Development Research Center of China Meteorological Administration. He mainly engages in fields of public management, public meteorological service, low carbon economy, fighting climate change etc.

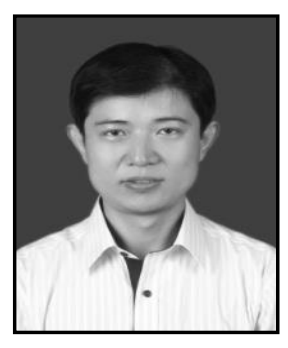

Dr. Jin Wang, received the B.S. and M.S. degree in the Electronical Engineering from Nanjing University of Posts and Telecommunications, China in 2002 and 2005, respectively. He received Ph.D. degree in the Ubiquitous Computing laboratory from the Computer Engineering Department of Kyung Hee University Korea in 2010. Now, he is a professor in the Computer and Software Institute, Nanjing University of Information Science and Technology. His research interests mainly include routing method and algorithm design, performance evaluation and optimization for wireless ad hoc and sensor networks. He is a member of the IEEE and ACM.

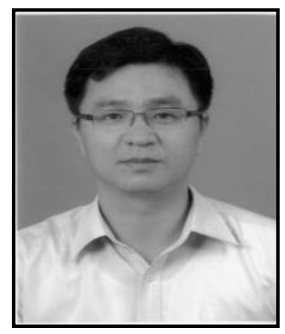

Dr. Jeong-Uk Kim, received his B.S. degree in Control and Instrumentation Engineering from Seoul National University in 1987, M.S. and Ph.D. degrees in Electrical Engineering from Korea Advanced Institute of Science and Technology in 1989, and 1993, respectively. He is a professor in SangMyung University in Seoul. His research interests include smart grid demand response, building automation system, and renewable energy. 